\title{
Maps stemming from the functional calculus that transform a Kubo-Ando mean into another
}

\author{
GERGÖ NAGY(D) \\ Dedicated to Professor János Aczél on the occasion of his ninety-fifth \\ birthday.
}

\begin{abstract}
In this paper, we investigate maps on sets of positive operators which are induced by the continuous functional calculus and transform a Kubo-Ando mean $\sigma$ into another $\tau$. We establish that under quite mild conditions, a mapping $\phi$ can have this property only in the trivial case, i.e. when $\sigma$ and $\tau$ are nontrivial weighted harmonic means and $\phi$ stems from a function which is a constant multiple of the generating function of such a mean. In the setting where exactly one of $\sigma$ and $\tau$ is a weighted arithmetic mean, we show that under fairly weak assumptions, the mentioned transformer property never holds. Finally, when both of $\sigma$ and $\tau$ are such a mean, it turns out that the latter property is only satisfied in the trivial case, i.e. for maps induced by affine functions.
\end{abstract}

Mathematics Subject Classification. Primary: 46L40; Secondary: 47L30.

Keywords. Homomorphisms, Mean affine functions, Functional equations, Kubo-Ando means, Continuous functional calculus, Positive operators, Invertible positive operators.

\section{Introduction and statement of the results}

Homomorphisms of algebraic structures form a basic object of study mainly in algebra, but they are highly relevant also in other areas of mathematics. In general, such transformations on structures of numbers can be described with certain functional equations. The most fundamental ones among them are the Cauchy equations, namely the additive, the multiplicative, the exponential and the logarithmic, which are related to the two most basic operations on real numbers, addition and multiplication. There is a vast literature devoted to them, we mention, e.g. the book of Aczél [1]. Their solutions were determined under very weak regularity assumptions, for example measurability. 
Homomorphisms are also considered between much more abstract structures than $\mathbb{R}$, e.g. between groups or algebras. For certain algebras, namely $C^{*}$-algebras, we can define continuous scalar functions also on some of their elements, namely the normal ones. Therefore, from the point of view of homomorphisms, one can consider morphism properties of transformations of the form $A \mapsto f(A)$, for example on the set $\mathcal{A}_{s}$ of self-adjoint elements of a $C^{*}$ algebra $\mathcal{A}$, where $f: \mathbb{R} \rightarrow \mathbb{R}$ is an unknown continuous function. This gives rise to a functional equation for $f$ on an abstract structure, e.g. $f(A+B)=$ $f(A)+f(B)\left(A, B \in \mathcal{A}_{s}\right)$. It is of the same form as its counterpart on $\mathbb{R}$, the additive Cauchy equation, however, here the value of a function at an element means the value of the continuous functional calculus for that element at the given function. Since the unknown functions in the $C^{*}$-algebra counterparts of the basic functional equations describing homomorphisms on $\mathbb{R}$ are necessarily continuous and these versions imply their validity also on $\mathbb{R}$, one can observe that their solutions can be obtained immediately, applying results on the form of continuous solutions of their scalar counterparts. Therefore their investigation is of no interest.

However, in some other cases, it can give rise to challenging functional equations. For the purposes of the paper, the most important among them is the case where the homomorphisms in question respect certain means, or in other words, transform a mean into another. These quantities form a fundamental concept in mathematics, originally they are introduced for the averaging of real numbers. A mean $M: D^{2} \rightarrow D$ on an interval $D \subset \mathbb{R}$ is defined as a binary operation satisfying the inequalities $\min \{x, y\} \leq M(x, y) \leq$ $\max \{x, y\} \quad(x, y \in D)$. Such objects have been intensively studied for a long time by many researchers, their investigation forms a broad field of mathematics. For details on means, the interested reader can consult, e.g. the short monograph [5] and the references therein. As for means of other objects, in [7] Kubo and Ando established the theory of operator means which are certain operations on the cone of positive operators on a Hilbert space. In the finite dimensional case, that notion reduces to means of positive semidefinite matrices which are widely used and investigated in several areas of mathematics.

To define those operator means, we introduce some necessary notation and definitions used throughout the paper. In what follows, $H$ denotes a complex Hilbert space with $\operatorname{dim} H \geq 2$. Let $B(H)$ stand for the $C^{*}$-algebra of bounded linear operators on $H$. An element $A \in B(H)$ is termed positive if $\langle A x, x\rangle \geq 0$ is satisfied by each vector $x \in H$ (which is the same as saying that it is a positive element of the $C^{*}$-algebra $\left.B(H)\right)$. Let $B(H)_{+}$, and $B(H)_{++}$denote the cone of positive operators, and invertible positive operators, resp. in $B(H)$. For a pair $A, B$ of self-adjoint elements in $B(H)$, we write $A \leq B$ whenever $B-A \in B(H)_{+}$. Finally, we denote by $I$, the identity operator on $H$. 
According to [7], we say that a binary operation $\sigma: B(H)_{+} \times B(H)_{+} \rightarrow$ $B(H)_{+}$is a Kubo-Ando mean if it possesses the properties below. For any elements $A, B, C, D \in B(H)_{+}$and sequences $\left(A_{n}\right),\left(B_{n}\right)$ in $B(H)_{+}$:

(i) $I \sigma I=I$ (normalization);

(ii) if $A \leq C$ and $B \leq D$, then $A \sigma B \leq C \sigma D$ (monotonicity);

(iii) $C(A \sigma B) C \leq(C A C) \sigma(C B C)$ (transfer property);

(iv) if $A_{n} \downarrow A$ and $B_{n} \downarrow B$, then $A_{n} \sigma B_{n} \downarrow A \sigma B$ (continuity).

Here, the symbol $\downarrow$ denotes monotone decreasing convergence in the strong operator topology. If $\sigma$ is a Kubo-Ando mean on $B(H)_{+}$, then its transpose $\tilde{\sigma}: B(H)_{+}^{2} \rightarrow B(H)_{+}$is defined by $A \tilde{\sigma} B=B \sigma A\left(A, B \in B(H)_{+}\right)$.

Kubo-Ando means can be represented by functions of a certain type which are defined as follows. A continuous map $f$ from a nontrivial interval $D \subset \mathbb{R}$ into $\mathbb{R}$ is called $n$-monotone (or matrix monotone of order $n \in \mathbb{N}$ ) if for each pair $A, B$ of self-adjoint operators on an $n$-dimensional complex Hilbert space whose spectra are in $D$, we have the implication $A \leq B \Rightarrow f(A) \leq f(B)$. We remark that such a function is obviously increasing. If $f$ is $n$-monotone for any integer $n \in \mathbb{N}$, then it is called operator monotone. Moreover, the continuous function $f: D \rightarrow \mathbb{R}$ is called $n$-concave if for any pair $A, B$ of operators with the above properties the inequality

$$
f(\alpha A+(1-\alpha) B) \geq \alpha f(A)+(1-\alpha) f(B) \quad(\alpha \in[0,1])
$$

is fulfilled.

From the verification of [7, Theorem 3.2], we get that for a Kubo-Ando mean $\sigma$ and a scalar $t \geq 0$ the operator $I \sigma(t I)$ is scalar. Hence, one can define a function $F_{\sigma}:[0, \infty[\rightarrow[0, \infty[$, referred to as the generating function of $\sigma$, satisfying

$$
F_{\sigma}(t) I=I \sigma(t I) \quad(t \geq 0) .
$$

Evidently, the property (i) yields $F_{\sigma}(1)=1$. The mentioned proof also shows that if $d=\operatorname{dim} H<\infty$, then $F_{\sigma}$ is $d$-monotone, otherwise it is operator monotone. Furthermore, $\sigma$ is a perspective mean, that is, it admits the explicit form

$$
A \sigma B=A^{1 / 2} f_{\sigma}\left(A^{-1 / 2} B A^{-1 / 2}\right) A^{1 / 2} \quad\left(A \in B(H)_{++}, B \in B(H)_{+}\right)
$$

and this, together with the property (iv), yields that $F_{\sigma}$ uniquely determines $\sigma$. Define $\tilde{F}_{\sigma}:=F_{\tilde{\sigma}}$. By $[7$, Corollary 4.2], we have

$$
\tilde{F}_{\sigma}(x)=x F_{\sigma}(1 / x) \quad(x>0) .
$$

Since Kubo-Ando means are operations on $B(H)_{+}$, a natural question arises: what are the homomorphisms of $B(H)_{+}$endowed with two Kubo-Ando means, more precisely, the maps $\phi: B(H)_{+} \rightarrow B(H)_{+}$satisfying that

$$
\phi(A \sigma B)=\phi(A) \tau \phi(B) \quad\left(A, B \in B(H)_{+}\right)
$$


for those means $\sigma, \tau: B(H)_{+}^{2} \rightarrow B(H)_{+}$? In other words, these are the mappings which transform $\sigma$ to $\tau$. The latter problem seems really challenging. As for related results, first we give the definitions of the three most important classes of Kubo-Ando means, the weighted arithmetic, geometric and harmonic means, whose generating functions on $] 0, \infty[$ are

$$
x \mapsto \alpha+(1-\alpha) x, x \mapsto x^{\alpha} \text { and } x \mapsto \frac{x}{\alpha x+1-\alpha} \quad(x>0,0 \leq \alpha \leq 1),
$$

moreover they are denoted by $\nabla_{\alpha}, \sharp_{\alpha}$ and $!_{\alpha}$, respectively. They have the explicit forms

$$
\begin{aligned}
A \nabla_{\alpha} B & =\alpha A+(1-\alpha) B, A \sharp_{\alpha} B=A^{\frac{1}{2}}\left(A^{-\frac{1}{2}} B A^{-\frac{1}{2}}\right)^{\alpha} A^{\frac{1}{2}}, \\
A !_{\alpha} B & =\left(\alpha A^{-1}+(1-\alpha) B^{-1}\right)^{-1}
\end{aligned}
$$

for all $A, B \in B(H)_{++}$. The structure of automorphisms of $B(H)_{+}$with respect to them was described in $[15,16]$ for $\alpha=1 / 2$. A result on the automorphisms of $B(H)_{+}$for any element of a large class of Kubo-Ando means can be found in [14], under some mild regularity assumption (e.g. a sort of continuity) on the transformations in question. As for theorems on maps transforming a Kubo-Ando mean into another, we refer to [13], where the previous three means are considered on the cone of invertible positive elements in a $C^{*}$-algebra (note that perspective means can be defined on such a cone).

Instead of determining the structure of all maps of $B(H)_{+}$transforming a Kubo-Ando mean into another, we can seek for only those which have a special form. Here, we consider maps of the form $A \mapsto f(A)\left(A \in B(H)_{+}\right)$ with some continuous function $f:[0, \infty[\rightarrow[0, \infty[$. Our aim is to establish equivalent conditions under which such a map transforms a Kubo-Ando mean into another. Mappings transforming a mean $M$ of real numbers into another one $N$ are usually termed $(M, N)$-affine, affine with respect to $M$ and $N$, or mean affine; they are investigated in, e.g. $[9,19]$. It is worth noting that $\left(\nabla_{1 / 2}, \nabla_{1 / 2}\right)$-affine functions are exactly the solutions of the well-known Jensen equation $f((x+y) / 2)=(f(x)+f(y)) / 2$, where $f$ is a real function. Adopting this terminology, for a pair of Kubo-Ando means $(\sigma, \tau)$ on $B(H)_{+}$, we say that a continuous function $f:[0, \infty[\rightarrow[0, \infty[$ is $(\sigma, \tau)$-affine, affine with respect to $\sigma$ and $\tau$ or mean affine if

$$
f(A \sigma B)=f(A) \tau f(B) \quad\left(A, B \in B(H)_{+}\right) .
$$

Observe that this property says that the map $A \mapsto f(A)\left(A \in B(H)_{+}\right)$transforms $\sigma$ into $\tau$. The aim of the paper is to investigate the last displayed functional equation on $B(H)_{+}$and $B(H)_{++}$. We remark that operator means on the cone of positive definite matrices of a given size are quite important in some areas of mathematics, e.g. they are intimately connected to certain differential geometric structures on those cones. Now our main result follows in which we consider the previous functional equation under some quite mild conditions. 
Theorem 1. Let $\sigma, \tau: B(H)_{+}^{2} \rightarrow B(H)_{+}$be Kubo-Ando means such that $F_{\sigma}, F_{\tau}$ are strictly concave and either $F_{\sigma}(0)=F_{\tau}(0)=0$ or $\tilde{F}_{\sigma}(0)=\tilde{F}_{\tau}(0)=0$. Moreover assume that $f:[0, \infty[\rightarrow[0, \infty[$ is a nonconstant continuous function for which $f(x)>0(x>0)$ and $f \neq \mathrm{id}$. Then the following statements are equivalent:

(i) $f(A \sigma B)=f(A) \tau f(B)$

(ii) $f(A \sigma B)=f(A) \tau f(B)$

$\left(A, B \in B(H)_{++}\right)$.

(iii) There exist numbers $\alpha, \beta>0 ; 0<\gamma<1$ such that

$$
f(x)=\frac{x}{\alpha+\beta x} \quad(x \geq 0)
$$

and $\sigma=\tau=!_{\gamma}$.

As for the conditions in this result, observe that a constant function $f$ satisfies (ii), and $f=$ id fulfills (i) or (ii) if and only if $\sigma=\tau$. Related to the concavity assumption, observe that the generating function of a KuboAndo mean on $B(H)_{+}$is $d$-monotone with an appropriate natural number $1<d \leq \operatorname{dim} H$. It is also concave by [8, Theorem 2.1] stating that any $d-$ monotone function on $] 0, \infty$ [ is concave of order $[d / 2]$. Observe that since $F_{\sigma}, F_{\tau}$ are strictly concave, they are positive on ]0, [, thus $\sigma, \tau$ are operations on $B(H)_{++}$. Moreover, the transformation $A \mapsto f(A)$ is a selfmap of $B(H)_{++}$. We remark that the condition $F_{\sigma}(0)=0$ means that $A \sigma 0=0$, while $\tilde{F}_{\sigma}(0)=0$ means $0 \sigma A=0\left(A \in B(H)_{+}\right)$. The result above shows the quite surprising fact that, under its conditions, a function is affine with respect to two Kubo-Ando means only in the trivial case.

In certain cases, the property that there exists a function which is affine with respect to two such means can be reformulated in another way. Concerning this, we recall the notion of a conjugated mean. For two means $M, N$ on an interval $D \subset \mathbb{R}$, we say that $N$ is a conjugated mean of $M$ if there exists a continuous injective function $f: D \rightarrow D$ with the property

$$
N(x, y)=f^{-1}(M(f(x), f(y))) \quad(x, y \in D) .
$$

We remark that the terminology refers to group theory. The most well-known examples of this concept are the quasi-arithmetic means which are the conjugated means of the arithmetic mean. Now a natural question arises: what are the conjugated means of a given one? Is it only itself? Or, in other words, can we get a new mean from a given one by conjugation? Of course, in the case of the arithmetic mean, the answer is affirmative, it is well-known that there are many quasi-arithmetic means. What about other means? First of all, observe that conjugated means can be defined also for Kubo-Ando means with the last displayed formula, where, in that case, $D=\left[0, \infty\left[\right.\right.$ and $x, y \in B(H)_{+}$. Moreover, as it can be seen easily, the right-hand side of that equation is well-defined for all $x, y \in B(H)_{+}$. Observe that a Kubo-Ando mean $\sigma$ is a conjugated mean of another one $\tau$ precisely when there exists an injective 
$(\sigma, \tau)$-affine function. In our next result, we investigate the conjugated means of a given one belonging to any of the sets

$$
\begin{aligned}
& \mathcal{M}_{1}=\left\{\sigma: B(H)_{+}^{2} \rightarrow B(H)_{+} \mid \sigma \text { is a Kubo-Ando mean, } F_{\sigma} \text { is strictly concave, } F_{\sigma}(0)=0\right\}, \\
& \mathcal{M}_{2}=\left\{\sigma: B(H)_{+}^{2} \rightarrow B(H)_{+} \mid \sigma \text { is a Kubo-Ando mean, } F_{\sigma} \text { is strictly concave, } \tilde{F}_{\sigma}(0)=0\right\} .
\end{aligned}
$$

Corollary. Let $i=1,2$ be a number and $\sigma \in \mathcal{M}_{i}$ be a Kubo-Ando mean. Then the only conjugated mean of $\sigma$ among the elements of $\mathcal{M}_{i}$ is $\sigma$.

As for the proof, observe that the existence of such a mean $M$ would imply the validity of (ii) in Theorem 1 for $M, \sigma$ and for the function $f$ appearing in the definition of conjugated means. It is also clear that $f, M, \sigma$ satisfy the conditions in that result (continuous injective functions are monotone), which would yield $M=\sigma$, a contradiction.

Observe that Theorem 1 covers two of the most fundamental classes of Kubo-Ando means, namely the sets of nontrivial weighted geometric and harmonic means, resp., but leave weighted arithmetic means untouched. To fill in this gap, in the next three results, we investigate the conditions under which (i) or (ii) in Theorem 1 holds for two Kubo-Ando means, at least one of them being a weighted arithmetic mean.

Theorem 2. Let $0<\alpha<1$ be a number and $\sigma: B(H)_{+}^{2} \rightarrow B(H)_{+}$be a KuboAndo mean such that either $F_{\sigma}(0)=0$ or $\tilde{F}_{\sigma}(0)=0$. Then there does not exist a nonconstant continuous function $f:[0, \infty[\rightarrow[0, \infty[$ such that $f(x)>0(x>$ $0)$ and

$$
f(A \sigma B)=f(A) \nabla_{\alpha} f(B) \quad\left(A, B \in B(H)_{++}\right) .
$$

Moreover, no nonconstant continuous function $f:[0, \infty[\rightarrow[0, \infty[$ satisfies this equality for all $A, B \in B(H)_{+}$.

Theorem 3. Let $0<\alpha<1$ be a number and $\sigma: B(H)_{+}^{2} \rightarrow B(H)_{+}$be a KuboAndo mean such that $F_{\sigma}$ is strictly concave and either $F_{\sigma}(0)=0$ or $\tilde{F}_{\sigma}(0)=0$. Then there does not exist a nonconstant continuous function $f:[0, \infty[\rightarrow[0, \infty[$ such that $f(x)>0(x>0)$ and

$$
f\left(A \nabla_{\alpha} B\right)=f(A) \sigma f(B) \quad\left(A, B \in B(H)_{++}\right) .
$$

Moreover, there are no nonconstant continuous functions $f:[0, \infty[\rightarrow[0, \infty[$ fulfilling this relation for each $A, B \in B(H)_{+}$.

The latter two theorems assert that for the members of a large class of Kubo-Ando means, there does not exist nonconstant functions which are affine with respect to one of them and to a nontrivial weighted arithmetic mean. Now our last result follows in which we establish that for means of the latter kind, the only mean affine functions are the trivial, affine ones. 
Theorem 4. Let $0<\alpha, \beta<1$ be numbers and $f:] 0, \infty[\rightarrow \mathbb{R}$ be a continuous function. Then $f$ fulfills

$$
f\left(A \nabla_{\alpha} B\right)=f(A) \nabla_{\beta} f(B) \quad\left(A, B \in B(H)_{++}\right)
$$

exactly when we have scalars $a, b \in \mathbb{R}$ such that $f(x)=a x+b(x>0)$ and in the case $a \neq 0$, the equality $\alpha=\beta$ holds.

It is worth mentioning that Theorem 3 says that there are no nontrivial weighted arithmetic means which are conjugated means of some element of $\mathcal{M}_{1} \cup \mathcal{M}_{2}$. As for the conjugation of other Kubo-Ando means, it follows from [17, Theorem 1.4.] that such an operation on $B(H)_{+}$is a weighted quasiarithmetic mean (i.e., a conjugated mean of a weighted arithmetic mean) exactly when it is a weighted arithmetic mean. In other words, the only KuboAndo means that are conjugated means of $\nabla_{\alpha}(0 \leq \alpha \leq 1)$ are the ones of this form, so only itself, by Theorem 4 .

\section{Proofs}

Rank-one (orthogonal) projections on $H$ will show up several times in this section, $P_{1}(H)$ stands for their class. The members of $P_{1}(H)$ are exactly the operators of the form $u \otimes u(u \in H,\|u\|=1)$, where the operation $\otimes$ is defined by

$$
u \otimes v: H \rightarrow H,(u \otimes v)(w):=\langle w, v\rangle u \quad(u, v, w \in H)
$$

In this section, we will use the well-defined quantity

$$
\lambda(A, P)=\left\langle A^{-1} u, u\right\rangle^{-1},
$$

where $A \in B(H)_{++}, P \in P_{1}(H)$ are elements and $u \in \operatorname{rng} P$ is a unit vector, moreover rng denotes the range of operators.

Now we are in a position to verify our main result.

Proof of Theorem 1. The implications (ii) $\Longrightarrow$ (i), (iii) $\Longrightarrow$ (ii) are trivial. Assume that (i) holds. In what follows, we show that, without loss of generality, $F_{\sigma}(0)=F_{\tau}(0)=0$ may be supposed. By [18, Lemma 1.3.2], a function $g$ from an interval $D \subset \mathbb{R}$ to $\mathbb{R}$ is strictly concave if and only if for any numbers $x_{1}<x_{2}<x_{3}$ in $D$, one has

$$
\operatorname{det}\left(\begin{array}{lll}
1 & x_{1} & g\left(x_{1}\right) \\
1 & x_{2} & g\left(x_{2}\right) \\
1 & x_{3} & g\left(x_{3}\right)
\end{array}\right)<0
$$


It can be checked easily that for any Kubo-Ando mean $\rho$ and each scalars $0<x_{1}<x_{2}<x_{3}$, the equality

$$
\frac{1}{x_{1} x_{2} x_{3}} \operatorname{det}\left(\begin{array}{lll}
1 & x_{1} & \tilde{F}_{\rho}\left(x_{1}\right) \\
1 & x_{2} & \tilde{F}_{\rho}\left(x_{2}\right) \\
1 & x_{3} & \tilde{F}_{\rho}\left(x_{3}\right)
\end{array}\right)=\operatorname{det}\left(\begin{array}{lll}
1 & 1 / x_{3} & f\left(1 / x_{3}\right) \\
1 & 1 / x_{2} & f\left(1 / x_{2}\right) \\
1 & 1 / x_{1} & f\left(1 / x_{1}\right)
\end{array}\right)
$$

holds. This gives us the strict concavity of $\tilde{F}_{\sigma}, \tilde{F}_{\tau}$ and then, since $\tilde{F}_{\sigma}=$ $F_{\tilde{\sigma}}, \tilde{F}_{\tau}=F_{\tilde{\tau}}$, it follows that the relation $F_{\sigma}(0)=F_{\tau}(0)=0$ can and will be assumed.

Next, observe that by (1), for any given Kubo-Ando mean $\rho: B(H)_{+}^{2} \rightarrow$ $B(H)_{+}$and operator $A \in B(H)_{++}$, the map $B \mapsto A \rho B\left(B \in B(H)_{+}\right)$is continuous in the metric induced by the operator norm and therefore (i) implies, referring to the well-known continuity of the map $T \mapsto f(T)\left(T \in B(H)_{+}\right)$,

$$
f(A \sigma B)=f(A) \tau f(B) \quad\left(A \in B(H)_{++}, B \in B(H)_{+}\right) .
$$

Furthermore if (ii) holds, then by the discussion in the previous paragraph, we may and do suppose that $F_{\sigma}(0)=F_{\tau}(0)=0$.

To sum up, we can conclude that both (i) and (ii) imply (5) and that $F_{\sigma}(0)=F_{\tau}(0)=0$ can and will be assumed. Hence it remains to show that (5) and the relations $F_{\sigma}(0)=F_{\tau}(0)=0$ yield (iii). To prove it, observe that from applying the property (iii) of Kubo-Ando means (see Sect. 1) their homogeneity follows, thus after multiplying (5) by $f(1)^{-1}$, wlog $f(1)=1$ may and will be supposed. Now using a proof by contradiction, we verify that $f(0)=0$. Since $f$ is not constant, there is a number $x_{0}>0$ for which $0<f\left(x_{0}\right) \neq f(0)$. Moreover, if $f(0) \neq 0$, then

$$
\begin{aligned}
& f(0) I=f\left(F_{\sigma}(0) I\right)=f(I \sigma 0)=f\left(\left(x_{0} I\right) \sigma 0\right) \\
& \quad=f\left(x_{0} I\right) \tau f(0)=f\left(x_{0}\right)\left(I \tau\left(\frac{f(0)}{f\left(x_{0}\right)} I\right)\right)=f\left(x_{0}\right) F_{\tau}\left(\frac{f(0)}{f\left(x_{0}\right)}\right) I
\end{aligned}
$$

which would imply $F_{\tau}\left(f(0) / f\left(x_{0}\right)\right)=f(0) / f\left(x_{0}\right)$. Since $F_{\tau}(x)=\operatorname{id}(x)$ for $x \in\{0,1\}$, furthermore $f(0) / f\left(x_{0}\right) \neq 0,1$; the second last equation would yield the equality of the strictly concave function $F_{\tau}$ and of an affine one at three distinct points, a contradiction.

We proceed by inserting an arbitrary element $B=P=u \otimes u(u \in H,\|u\|=$ 1) in (5). To get the desired conclusion from this substitution, we need a formula for $A \rho P$, where $A \in B(H)_{++}$is an operator and $\rho$ is a Kubo-Ando mean with $F_{\rho}(0)=0$. This can be derived by the computation

$$
\begin{aligned}
& A \rho P=A^{1 / 2} F_{\rho}\left(A^{-1 / 2} u \otimes u A^{-1 / 2}\right) A^{1 / 2} \\
& \quad=F_{\rho}\left(\left\|A^{-1 / 2} u\right\|^{2}\right) A^{1 / 2}\left(\left(1 /\left\|A^{-1 / 2} u\right\|\right) A^{-1 / 2} u\right) \otimes\left(\left(1 /\left\|A^{-1 / 2} u\right\|\right) A^{-1 / 2} u\right) A^{1 / 2} \\
& =\frac{F_{\rho}\left(\left\|A^{-1 / 2} u\right\|^{2}\right)}{\left\|A^{-1 / 2} u\right\|^{2}} P=\lambda(A, P) F_{\rho}\left(\frac{1}{\lambda(A, P)}\right) P=\tilde{F}_{\rho}(\lambda(A, P)) P,
\end{aligned}
$$


from which we conclude that $A \rho P=\tilde{F}_{\rho}(\lambda(A, P)) P$. Now applying (5) for $B=P$ and this formula (observe that $A \in B(H)_{++}$and $f(x)>0(x>0)$, so $\left.f(A) \in B(H)_{++}\right)$, because of the equalities $f(0)=0, f(1)=1$, it follows that $f\left(\tilde{F}_{\sigma}(\lambda(A, P))\right) P=\tilde{F}_{\tau}(\lambda(f(A), P)) P$, i.e.

$$
f\left(\tilde{F}_{\sigma}(\lambda(A, P))\right)=\tilde{F}_{\tau}(\lambda(f(A), P)) .
$$

Next put $A=x I, B=y I(x, y>0)$ in (5) to get the relation

$$
f\left(x F_{\sigma}\left(\frac{y}{x}\right)\right)=f(x) F_{\tau}\left(\frac{f(y)}{f(x)}\right)
$$

which, for $y=1$, gives us that $f\left(\tilde{F}_{\sigma}(x)\right)=\tilde{F}_{\tau}(f(x))$. This together with (6) implies

$$
\tilde{F}_{\tau}(f(\lambda(A, P)))=\tilde{F}_{\tau}(\lambda(f(A), P)) .
$$

Observe that since $\tilde{F}_{\tau}$ is strictly concave and monotone, it is injective. This fact together with the last displayed equality gives us that $f(\lambda(A, P))=$ $\lambda(f(A), P)$.

It implies

$$
f\left(\left\langle A^{-1} u, u\right\rangle^{-1}\right)=\left\langle f(A)^{-1} u, u\right\rangle^{-1} .
$$

Now let $e_{1}, e_{2} \in H ; x, y>0 ; 0<\mu<1$ be arbitrary elements such that $e_{1}, e_{2}$ are mutually orthogonal unit vectors, and plug

$$
\begin{aligned}
& A=x \cdot e_{1} \otimes e_{1}+y \cdot e_{2} \otimes e_{2}+I-\left(e_{1} \otimes e_{1}+e_{2} \otimes e_{2}\right), \\
& P=\left(\sqrt{\mu} e_{1}+\sqrt{1-\mu} e_{2}\right) \otimes\left(\sqrt{\mu} e_{1}+\sqrt{1-\mu} e_{2}\right)
\end{aligned}
$$

in the previous equality to obtain, using the basic properties of functional calculus, that

$$
f\left(\left(\mu x^{-1}+(1-\mu) y^{-1}\right)^{-1}\right)=\left(\mu f(x)^{-1}+(1-\mu) f(y)^{-1}\right)^{-1} .
$$

In the rest of the proof, for any function $g:[0, \infty[\rightarrow[0, \infty[$ with $g(x)>0(x>$ $0)$, let $\left.\hat{g}=\left.(1 / \mathrm{id}) \circ g\right|_{] 0, \infty[} \circ(1 / \mathrm{id}):\right] 0, \infty[\rightarrow] 0, \infty[$. Applying the last displayed equality, it is very easy to see that for all $r, s>0$, one has $\hat{f}(\mu r+(1-\mu) s)=$ $\mu \hat{f}(r)+(1-\mu) \hat{f}(s)$ showing that $\hat{f}$ is affine. Hence, by the fact that $\hat{f}(x)>0$, there are numbers $\alpha, \beta \geq 0$ not both 0 such that $\hat{f}(x)=\alpha x+\beta$, i.e. $f(x)=$ $\left(\alpha x^{-1}+\beta\right)^{-1}$. Then from the assumptions that $f$ is not constant, $f(1)=1, f \neq$ id; we infer that $\alpha+\beta=1,0<\beta<1$ and thus $f(x)=x /(1-\beta+\beta x)$.

To complete the proof, we have to show that $F_{\sigma}, F_{\tau}$ are of the form appearing in (iii). To do this, let $x, y>0$ be arbitrary numbers and substitute the latter form of $f$ in (7) in order to get that

$$
\frac{x F_{\sigma}\left(\frac{y}{x}\right)}{1-\beta+\beta x F_{\sigma}\left(\frac{y}{x}\right)}=\frac{x}{1-\beta+\beta x} F_{\tau}\left(\frac{1-\beta+\beta x}{1-\beta+\beta y} \frac{y}{x}\right) .
$$


Now, as it was already mentioned, if $f, F_{\sigma}, F_{\tau}$ have the forms appearing in (iii), then (ii) holds. This suggests that $F_{\sigma}, F_{\tau}$ should be of those forms if (ii) is satisfied. But if they are so, then $\hat{F}_{\sigma}, \hat{F}_{\tau}$ are affine, so, using the last displayed relation, we aim to get an equality which involves a convex combination of values of these functions and their values at a convex combination. The mentioned relation implies

$$
\hat{F}_{\tau}\left(\frac{1-\beta+\beta y}{1-\beta+\beta x} \frac{x}{y}\right)=\frac{(1-\beta) \hat{F}_{\sigma}\left(\frac{x}{y}\right)+\beta x}{1-\beta+\beta x},
$$

where the left-hand side equals

$$
\hat{F}_{\tau}\left(\frac{(1-\beta) \frac{x}{y}+\beta x}{1-\beta+\beta x}\right),
$$

therefore, with the notation $t=1 / y$, the latter equality yields that

$$
\hat{F}_{\tau}\left(\frac{(1-\beta)(x t)+\beta x}{(1-\beta)+\beta x}\right)=\frac{(1-\beta) \hat{F}_{\sigma}(x t)+\beta x}{(1-\beta)+\beta x} .
$$

Next, let $0<\nu<1$ be an arbitrary number. It is clear that we can find a scalar $x_{\nu}>0$ for which $\nu=(1-\beta) /\left((1-\beta)+\beta x_{\nu}\right)$. Then it follows that for all $t>0$, the equation $\hat{F}_{\tau}\left(\nu\left(x_{\nu} t\right)+1-\nu\right)=\nu \hat{F}_{\sigma}\left(x_{\nu} t\right)+1-\nu$ is valid entailing that

$$
\hat{F}_{\tau}(\nu s+1-\nu)=\nu \hat{F}_{\sigma}(s)+1-\nu, \quad \forall 0<\nu<1,0<s .
$$

Theorem 2.4.7. in [6] tells us that $n$-monotone functions on open bounded intervals are in $C^{2 n-3}(n \in \mathbb{N} \backslash\{1\})$, giving us that the $d$-monotone generating functions $F_{\sigma}, F_{\tau}$ are in $C^{1}(] 0, \infty[)$

( $\operatorname{dim} H>1$ !), where $1<d \leq \operatorname{dim} H$ is an appropriate natural number. Hence $\hat{F}_{\sigma}, \hat{F}_{\tau} \in C^{1}(] 0, \infty[)$, and thus differentiating (8) with respect to $\nu$, we arrive at the relation $(s-1) \hat{F}_{\tau}^{\prime}(\nu s+1-\nu)=\hat{F}_{\sigma}(s)-1$ for any given $s>0$. By the latter facts, we can take the limit $\nu \rightarrow 0$ in this equality and then obtain that $(s-1) \hat{F}_{\tau}^{\prime}(1)=\hat{F}_{\sigma}(s)-1$, from which we deduce the relation $\hat{F}_{\sigma}(s)=\gamma s+1-\gamma$ with $\gamma=\hat{F}_{\tau}^{\prime}(1)$. Now we conclude that $F_{\sigma}(x)=$ $\left(\gamma x^{-1}+1-\gamma\right)^{-1}(x>0)$ and, because of the conditions on $F_{\sigma}$, the inequalities $0<\gamma<1$ follow. Finally, substituting the previous form of $\hat{F}_{\sigma}$ in (8) and passing to the limit $\nu \rightarrow 1$ in the relation obtained so, we arrive at the formula $\hat{F}_{\tau}(s)=\gamma s+1-\gamma(s>0)$ entailing the desired equality $F_{\tau}=F_{\sigma}$. By what we have proved so far and having in mind how we have reached the assumptions $F_{\sigma}(0)=F_{\tau}(0)=0, f(1)=1$; the condition (iii) follows and this completes the proof of Theorem 1 .

Proof of Theorem 2. We can follow the argument in the first two paragraphs of the previous proof to show that under the conditions of Theorem 2, if (2) is satisfied by all $A, B \in B(H)_{++}$and by a nonconstant continuous function 
$f:\left[0, \infty\left[\rightarrow\left[0, \infty\left[\right.\right.\right.\right.$ with $f(x)>0(x>0)$, then it holds for each $B \in B(H)_{+}$; and we may and do suppose the validity of $F_{\sigma}(0)=0$. Moreover, this assumption can and will be used also in the case where $f$ is not supposed to have the property $f(x)>0(x>0)$ but it fulfills (2) for any $A, B \in B(H)_{+}$. Thus in both cases, we have that $F_{\sigma}(0)=0$ and $f:[0, \infty[\rightarrow[0, \infty[$ is a nonconstant continuous function satisfying (2) for every $A \in B(H)_{++}, B \in B(H)_{+}$. Let $A \in B(H)_{++}$be an operator and apply (2) to $B=0$ in order to obtain, using $F_{\sigma}(0)=0$, that $f(0) I=\alpha f(A)+(1-\alpha) f(0) I$, i.e. $f(A)=f(0) I$. Since $f$ is not constant, there is a number $x_{0}>0$ for which $f\left(x_{0}\right) \neq f(0)$. Now by putting $A=x_{0} I$ in the last equality, we arrive at a contradiction and then we can conclude that the proof of Theorem 2 is complete.

Proof of Theorem 3. In the same way as in the previous argument, we get that if $f$ satisfies the conditions either in the second or in the third sentence of Theorem 3, then the equality (3) holds for all $A \in B(H)_{++}, B \in B(H)_{+}$, and we may and do suppose the validity of $F_{\sigma}(0)=0$. Of course, we also have that $f:[0, \infty[\rightarrow[0, \infty[$ is a nonconstant continuous function in both cases. Then, similarly to the third paragraph of the first proof, one can deduce the equality $f(0)=0$. Next, pick an operator $A \in B(H)_{++}$and insert $B=0$ in (3) to get that $f(\alpha A)=0$. Since $f$ is continuous and not constant, we can find a number $x_{0}>0$ for which $f\left(x_{0}\right) \neq 0$ and then it follows that by putting $A=\left(x_{0} / \alpha\right) I$ in the previous equality, we arrive at $f\left(x_{0}\right) I=0$, a contradiction. Finally, we obtain the conclusions in Theorem 3.

Proof of Theorem 4. Let $x, y>0$ be numbers and insert $A=x I, B=y I$ in (4) to obtain that $f(\alpha x+(1-\alpha) y)=\beta f(x)+(1-\beta) f(y)$ implying $f(s+t)=$ $g(s)+h(t)(s, t>0)$ for the functions

$$
g=(\beta \cdot \mathrm{id}) \circ f \circ((1 / \alpha) \cdot \mathrm{id}), h=((1-\beta) \cdot \mathrm{id}) \circ f \circ((1 /(1-\alpha)) \cdot \mathrm{id}) .
$$

This means that $f, g, h$ satisfy the Pexiderized Cauchy additive functional equation on the domain $\emptyset \neq] 0, \infty[\times] 0, \infty\left[\subset \mathbb{R}^{2}\right.$, therefore Theorem 4 in $[1$, p. 80] applies yielding the existence of a function $\varphi: \mathbb{R} \rightarrow \mathbb{R}$ and constants $b_{1}, b_{2} \in \mathbb{R}$ with the properties that $\varphi$ fulfills the Cauchy additive equation and $f=\varphi+b_{1}+b_{2}, g=\varphi+b_{1}, h=\varphi+b_{2}$. However, the continuity of $f$ implies that of $\varphi$ and thus we immediately obtain that one can find a number $a \in \mathbb{R}$ for which $f(x)=a x+b_{1}+b_{2}=((a \alpha) / \beta) x+b_{1} / \beta$, hence $a=a(\alpha / \beta)$, and if $a \neq 0$, then $\alpha=\beta$. The statement of Theorem 4 follows.

\section{Concluding remarks}

As far as we know, the structure of homomorphisms of $B(H)_{+}$or $B(H)_{++}$ with respect to a Kubo-Ando mean is still unknown in full generality even in the bijective case. Here we have obtained partial results about this problem 
which are quite general but only relative to the setting of homomorphisms induced by the continuous functional calculus.

Observe also that the scalar case is missing from those theorems. In that case, (i) in Theorem 1 implies that (7) holds for all $x, y>0$, which is a functional equation for the unknown functions $f, F_{\sigma}, F_{\tau}$, where, in general, $f:\left[0, \infty\left[\rightarrow\left[0, \infty\left[\right.\right.\right.\right.$ is continuous, $f(x)>0(x>0)$ and $F_{\sigma}, F_{\tau}$ are generating functions of Kubo-Ando means on $[0, \infty[$. It is obvious that a function $G:[0, \infty[\rightarrow[0, \infty[$ of the latter kind has the properties that

$$
G \text { is continuous; } G, \tilde{G} \text { are increasing; and } G(1)=1 \text {. }
$$

Now suppose that a function $G:[0, \infty[\rightarrow[0, \infty[$ satisfies these conditions. Then one can easily check that the operation $\rho_{G}:\left[0, \infty\left[{ }^{2} \rightarrow[0, \infty[\right.\right.$ defined by the formula

$$
\rho_{G}(a, b)=\left\{\begin{array}{ll}
a G\left(\frac{b}{a}\right) & \text { if } a \neq 0 \\
\tilde{G}(0) b & \text { if } a=0
\end{array} \quad(a, b \in[0, \infty[)\right.
$$

is a Kubo-Ando mean. Finally, we conclude that the generating functions of Kubo-Ando means on $[0, \infty[$ are exactly the maps $G:[0, \infty[\rightarrow[0, \infty[$ fulfilling the conditions in (9). In what follows, we mention two immediate consequences of this assertion.

The first one is that the Kubo-Ando means on $[0, \infty[$ are precisely the continuous monotone homogeneous means on $[0, \infty[$. Indeed, the normalization property of such means coincides with the property that they are reflexive as usual means of real numbers, and their transfer property is equivalent to their homogeneity. Moreover, we mention that if $D \subset \mathbb{R}$ is an interval, then every normalized increasing function is a mean (see [10, Remark 3]). The converse implication is false.

The second consequence of the last assertion in the last but one paragraph is that in the one dimensional case, (i) in Theorem 1 is equivalent to a functional equation of the form

$$
f\left(x g\left(\frac{y}{x}\right)\right)=f(x) h\left(\frac{f(y)}{f(x)}\right) \quad(x, y>0),
$$

where for the unknown functions $f, g, h:[0, \infty[\rightarrow[0, \infty[$, one has that $f$ is continuous, $f(x)>0(x>0)$ and $g$, $h$ satisfy the properties in (9). We highlight that, by the preceding discussion, each $\left(\rho_{g}, \rho_{h}\right)$-affine function is a solution of this equation, i.e. any map which is affine with respect to two continuous monotone homogeneous means on $[0, \infty[$. Functions $f:] 0, \infty[\rightarrow] 0, \infty[$ that are affine with respect to $M, M$, where $M$ is a homogenenous mean on $] 0, \infty[$ are investigated in [9]. There, analogously to Theorem 1, it was shown that, under some conditions, an $(M, M)$-affine function which is also continuous at a point and neither constant, nor linear exists only when $M$ is of a certain type, namely 
a weighted power mean, i.e., a map of the form

$$
(x, y) \mapsto\left(\alpha x^{p}+(1-\alpha) y\right)^{1 / p} \quad(x, y>0 ; p \in \mathbb{R} \backslash\{0\} ; 0 \leq \alpha \leq 1) .
$$

One can also consider the inequality versions of the relation defining mean affine functions, i.e.,

$$
f(M(x, y)) \leq N(f(x), f(y)) \quad(x, y \in D)
$$

and the reversed inequality, where $f: D \rightarrow E$ is a function and $M$, and $N$ are means on the intervals $D$, and $E$ in $\mathbb{R}$, resp. The functions $f: D \rightarrow E$ satisfying (10) (the reverse of $(10))$ are called $(M, N)$-convex $((M, N)$-concave) or convex (concave) with respect to $M, N$ (see $[2,4,9,11,12])$. Clearly, the functions which are convex (concave) with respect to $\nabla_{\alpha}, \nabla_{\alpha}$ for all $\alpha \in[0,1]$ are exactly the convex (concave) ones and the $\left(\nabla_{1 / 2}, \nabla_{1 / 2}\right)$-convex $\left(\left(\nabla_{1 / 2}, \nabla_{1 / 2}\right)\right.$-concave $)$ functions are precisely the Jensen-convex (Jensen-concave) ones.

Analogously to the case of affinity, convex functions with respect to means can be defined also in the case of Kubo-Ando means using (10), where, in that case, $M, N$ are such operations on $B(H)_{+}$, moreover $f:[0, \infty[\rightarrow[0, \infty[$ is a continuous function and $x, y \in B(H)+$ are arbitrary operators. For such objects $M, N, f$, we say that $f$ is $(M, N)$-convex $((M, N)$-concave) or convex (concave) with respect to $M, N$, if it satisfies (10) (the reverse of (10)) for all $x, y \in B(H)_{+}$. Observe that in the case $\operatorname{dim} H=n(n \in \mathbb{N})$, the functions which are concave with respect to $\nabla_{\alpha}, \nabla_{\alpha}$ (as means on $B(H)_{+}$) for each $\alpha \in[0,1]$ are exactly the $n$-concave ones on $[0, \infty$ [ (see Sect. 1 . for the definition of the latter functions). We remark that a real-valued continuous function $f$ on a nontrivial interval $D \subset \mathbb{R}$ is called $n$-convex if $-f$ is $n$-concave. The functions which are $n$-convex ( $n$-concave) for any $n \in \mathbb{N}$ are termed operator convex (concave). Functions of the latter four kinds appear, e.g. in the theory of operator means and quantum divergences. Furthermore, it is known that operator convex and concave functions have a certain integral representation.

As for convex/concave functions with respect to general Kubo-Ando means, we refer to Theorem 2.1. (2.3.) in [3] stating that in the case where $\operatorname{dim} H=\infty$, for such a symmetric mean $\sigma \neq \nabla_{1 / 2}\left(\sigma \neq !_{1 / 2}\right)$ on $B(H)_{+}$, if a continuous function $f:\left[0, \infty\left[\rightarrow\left[0, \infty\left[\right.\right.\right.\right.$ is $\left(\nabla_{1 / 2}, \sigma\right)$-convex $\left(\left(\nabla_{1 / 2}, \sigma\right)\right.$-concave $)$, then $-f$ $(f)$ is operator monotone. We believe that it is a really challenging problem to obtain results on the general forms of convex/concave functions with respect to Kubo-Ando means. Our conjecture is that under not too strong conditions, they have very special forms. 


\section{Acknowledgements}

Open access funding provided by University of Debrecen. The author was supported by the National Research, Development and Innovation Office NKFIH Reg. No. K115383.

Open Access. This article is licensed under a Creative Commons Attribution 4.0 International License, which permits use, sharing, adaptation, distribution and reproduction in any medium or format, as long as you give appropriate credit to the original author(s) and the source, provide a link to the Creative Commons licence, and indicate if changes were made. The images or other third party material in this article are included in the article's Creative Commons licence, unless indicated otherwise in a credit line to the material. If material is not included in the article's Creative Commons licence and your intended use is not permitted by statutory regulation or exceeds the permitted use, you will need to obtain permission directly from the copyright holder. To view a copy of this licence, visit http:// creativecommons.org/licenses/by/4.0/.

Publisher's Note Springer Nature remains neutral with regard to jurisdictional claims in published maps and institutional affiliations.

\section{References}

[1] Aczél, J.: A Short Course on Functional Equations. D. Reidel Publishing Company, Dordrecht (1987)

[2] Aczél, J.: A generalization of the notion of convex functions. Norske Vid. Selsk. Forh., Trondhjem 19, 87-90 (1947)

[3] Ando, T., Hiai, F.: Operator log-convex functions and operator means. Math. Ann. 350, 611-630 (2011)

[4] Aumann, G.: Konvexe Funktionen und Induktion bei Ungleichungen zwischen Mittelwerten, S.-B. math.-naturw. Abt. Bayer. Akad. Wiss. München no. III , 405-413 (1933)

[5] Daróczy, Z., Páles, Zs: Gauss-composition of means and the solution of the MatkowskiSutô problem. Publ. Math. Debrecen 61, 157-218 (2002)

[6] Hiai, F.: Matrix analysis: matrix monotone functions, matrix means, and majorization. Interdiscip. Inf. Sci. 16, 139-248 (2010)

[7] Kubo, F., Ando, T.: Means of positive linear operators. Math. Ann. 246, 205-224 (1980)

[8] Mathias, R.: Concavity of monotone matrix functions of finite order. Linear Multilinear Algebra 27, 129-138 (1990)

[9] Matkowski, J.: Convex functions with respect to a mean and a characterization of quasiarithmetic means. Real Anal. Exchange 29, 229-246 (2003/04)

[10] Matkowski, J.: Iterations of the mean-type mappings. In: Iteration Theory (ECIT'08), A.N. Sharkovsky, I.M. Sushko (Eds.), Grazer Math. Ber., Bericht Nr. 354, pp. 158-179, Fachbibliothek für Math., Karl-Franzens-University (2009)

[11] Matkowski, J., Rätz, J.: Convexity of power functions with respect to symmetric homogeneous means. Internat. Ser. Numer. Math. 123, 231-247 (1997)

[12] Matkowski, J., Rätz, J.: Convex functions with respect to an arbitrary mean. Internat. Ser. Numer. Math. 123, 249-259 (1997)

[13] Molnár, L.: The arithmetic, geometric and harmonic means in operator algebras and transformations among them. In: Recent Methods and Research Advances in Operator Theory, F. Botelho, R. King, T.S.S.R.K. Rao (Eds.) Contemporary Mathematics, Vol. 687, pp. 182-193, American Mathematical Society (2017) 
[14] Molnár, L.: Maps preserving general means of positive operators. Electron. J. Linear Algebra 22, 864-874 (2011)

[15] Molnár, L.: Maps preserving the harmonic mean or the parallel sum of positive operators. Linear Algebra Appl. 430, 3058-3065 (2009)

[16] Molnár, L.: Maps preserving the geometric mean of positive operators. Proc. Amer. Math. Soc. 137, 1763-1770 (2009)

[17] Nagy, G., Szokol, P.: Maps preserving norms of generalized weighted quasi-arithmetic means of invertible positive operators. Electron. J. Linear Algebra 35, 357-364 (2019)

[18] Niculescu, C., Persson, L.-E.: Convex Functions and Their Applications. A Contemporary Approach. CMC Books in Mathematics, Springer, New York (2006)

[19] Nowak, A.: On affine functions with respect to some means. Aequationes Math. 80, 269-275 (2010)

Gergő Nagy

Institute of Mathematics

University of Debrecen

4002 Debrecen P.O. Box 400

Hungary

e-mail: nagyg@science.unideb.hu

Received: December 15, 2019

Revised: July 7, 2020 